Shari Oesterreich, MD

Resident, Center for Anesthesiology Education,

Anesthesiology Institute, Cleveland Clinic
Jacek B. Cywinski, MD, FASA

Department of General Anesthesiology,

Anesthesiology Institute, Cleveland Clinic
Brett Elo, DO

Department of Intensive Care and Resuscitation,

Anesthesiology Institute, Cleveland Clinic

Mariya Geube, MD

Department of Cardiothoracic Anesthesiology,

Piyush Mathur, MD, FCCM

Department of General Anesthesiology,

Anesthesiology Institute, Cleveland Clinic

\title{
Quality improvement during the COVID-19 pandemic
}

\section{Posted May 29, 2020}

\section{ABSTRACT}

In response to the COVID-19 pandemic, quality improvement teams at Cleveland Clinic initiated a number of measures to guide the care of patients with suspected or confirmed COVID-19 infection and protect care givers. This included increasing the frequency of team meetings from monthly to daily or weekly and creating task forces to create protocols for patient transport, airway management, and management of personal protective equipment and medications in short supply. Enterprise wide, we postponed non-essential surgeries, set up an overflow intensive care unit onsite, created a web-based COVID-19 toolkit for all care givers, and sent daily emails about the most recent developments, decisions, and recommendations from national and international societies.

\section{INTRODUCTION}

The COVID-19 pandemic has presented unique challenges to quality improvement within health care institutions. Beyond maintaining established standards and ensuring compliance with best practice models, quality teams at Cleveland Clinic were tasked to innovate, adapt, and develop new guidelines to guide the care of patients with suspected or confirmed COVID-19 infection as well as protect caregivers. We share our experience managing various aspects of quality improvement during the initial months of the pandemic.

The statements and opinions expressed in COVID-19 Curbside Consults are based on experience and the available literature as of the date posted. While we try to regularly update this content, any offered recommendations cannot be substituted for the clinical judgment of clinicians caring for individual patients.

doi:10.3949/ccjm.87a.ccc041

\section{QUALITY IMPROVEMENT COMMITTEE STRUCTURE AND WORKINGS}

The structure and function of the quality improvement committee were enhanced during the onset of the COVID-19 pandemic. The frequency of our meetings was increased from monthly to weekly or daily huddles, depending on the needs of the different institutes. Quality committees provided caregivers throughout the enterprise with specific skills and expertise needed to perform new tasks aligned with their particular fields of interest. During meetings, team members discussed leadership updates, changes in policies, and new standard operating procedures. Time was also allocated to discuss new issues related to the changing environment.

In an integrated structure, task forces were created to design various protocols and documents for review and approval by members of the quality control committee, which were ultimately distributed throughout the institution. Topics and scenarios included patient transport, airway management guidelines, and management of personal protective equipment (PPE) and medications in short supply. These newly created protocols and documents allowed the quality committee to provide up-to-date guidance based on available best practices as well as standardize management and workflow across the different institutes. ${ }^{1-3}$

\section{MEASURING DATA}

It is important to report quality and patient safety data for both COVID-19-related and unrelated events. Pre-existing quality metrics such as severe harm event rates, surgical site infection rates, and safety event reports need continued measurement, although capturing data for in-person assessments, such as compliance with hand hygiene or checklists, may require a different mechanism to evaluate.

Additional metrics and data should be reviewed to understand not only the impact of the current pandemic on quality of care but also to assess caregiver 
safety. Occupational health, infection prevention, and other data sources can also be leveraged to assess the impact of COVID-19.2,4

\section{POLICY AND PROCEDURAL CHALLENGES}

Preparation of new policies and procedures during a pandemic, or modification of existing ones, requires changes to the standard approach. While certain policies still require approval using predefined processes, modifications or approvals of those related to the pandemic require a new process for rapid cycle approval. Creating live documents that reside within an institution's intranet and can be updated based on evolving recommendations is an important and practical solution that allows all caregivers to have access to the most up-to-date recommendations

Exemptions for various regulatory processes have been provided by organizations such as the Centers for Medicare and Medicaid Services and The Joint Commission. It is still important to comply with the policies and procedures that are not affected by the change, although additional efforts may be needed to refocus prior top safety concerns and countermeasures. $2,3,5$

\section{ENTERPRISE INTEGRATION}

In order to present a united front and support our community in the best possible manner during this pandemic, it has been extremely important for enterprise quality representatives, surgical services, community hospital liaisons, and department leaders to come together quickly for combined decision making in an integrated structure. Working together in this fashion facilitated rapid internal mobilization and unified response that spanned the enterprise.

We worked as a multifaceted team to innovate in both clinical and research settings, respond rapidly to change as more information was uncovered, and develop effective policies and procedures that balanced the need to contain the spread of COVID-19 with our organization's priority to ensure that all patients continue to receive best-in-class care.

Actions taken included postponing non-essential surgeries, setting up an overflow intensive care unit (ICU) on-site as well as related deployment teams and rotating schedules to be used in case of a surge, and creating a staggered plan for slowly returning to normal hospital activity once the pandemic started to level out. $2,3,5$

\section{EDUCATION AND IMPLEMENTATION}

A multifaceted approach was used to inform the caregivers about clinical considerations and the current status of the COVID-19 pandemic. A web-based
COVID-19 toolkit was made easily accessible from the Cleveland Clinic provider homepage, containing quick links to detection protocols, PPE recommendations, precautionary guidelines, care considerations, and a number of other educational and reference materials. A SharePoint site was established to collaborate on and maintain ICU-specific workflows and guidelines.

Special attention was paid to creating an ICU training curriculum to educate non-ICU providers with different backgrounds on how to care for critically ill patients (including ventilator management, nutrition, and documentation and billing procedures). Online MyLearning modules were created to facilitate training and education.

Daily emails were sent at both the enterprise and departmental levels to inform caregivers and support staff about the most recent developments, decisions, and recommendations. In addition to inward-focused communication, an external-facing website was created to inform the local community about safety measures they can take and how to screen themselves before calling in for an appointment. All of these measures have worked together to disseminate clear and consistent information to providers and the public. Quality education for trainees, both within the institute and the organization, continued virtually in order to maintain social distancing. . $^{2,46-8}$

\section{NATIONAL SOCIETY GUIDELINES AND RECOMMENDATIONS}

The healthcare field is fortunate to have expert guidance from major national and international societies such as the US Centers for Disease Control and Prevention and the World Health Organization. Our diverse institution, which is composed of various types of caregivers, is also influenced by many specialty-specific societies. The pandemic presented a dynamic situation, often bringing forth new information and knowledge on a daily basis. As experience and evidence changed, so did the recommendations and guidelines of the many societies.

Along with the plethora of societies came a diverse set of recommendations, oftentimes differing from one society to the next. The quality committee was able to review all of the relevant recommendations and guidelines. Our goal was to provide our institution with the best practices for our patients and the safest protection for our caregivers. While making these policies, the committee considered both the impact of COVID-19 on our specific geographical location as well as the status of our institution's equipment, material, drug, and personnel supply., $2,4-11$ 


\section{CONCLUSION}

Quality improvement during a pandemic requires a dynamic, rapid cycle, plan-do-check-act cycle approach. Leadership at all levels is key to coordinate, develop protocols and implement change to sustain quality improvement during disasters such as pandemics.

\section{KEY RECOMMENDATIONS}

1. Structural and procedural changes need to be made to the quality teams to adapt to a dynamic pandemic environment.

2. Engagement and communication are key both within the quality teams and with external leadership groups.

3. The timely delivery of recommendations to clinicians with a multimodal approach is important.

4. It is important to continue safety monitoring and enhance reporting structures to capture pandemic-associated safety events.

5. The focus should remain on both pandemicrelated quality metrics and pre-existing ones.

\section{REFERENCES}

1. Cohen MM, Eustis MA, Gribbins RE. Changing the culture of patient safety: leadership's role in health care quality improve- ment. Jt Comm J Qual Saf 2003; 29(7):329-335. doi:10.1016/ s1549-3741(03)29040-7

2. Wong J, Goh QY, Tan Z, et al. Preparing for a COVID-19 pandemic: a review of operating room outbreak response measures in a large tertiary hospital in Singapore. Can J Anaesth 2020; 67(6):732-745. doi:10.1007/s12630-020-01620-9

3. Wu AW, Connors C, Everly GS Jr. COVID-19: peer support and crisis communication strategies to promote institutional resilience. Ann Intern Med 2020; April 6. doi:10.7326/M20-1236

4. Sonis JD, Black L, Baugh J, et al. Leveraging existing quality improvement communication strategies during the COVID-19 crisis: creation of an emergency department COVID-19 case conference. Am J Emerg Med 2020; April 11. doi:10.1016/j.ajem.2020.04.021

5. Gilat R, Haunschild ED, Tauro T, Cole BJ. Recommendation to optimize safety of elective surgical care while limiting the spread of COVID-19: Primum Non Nocere. Arthrosc Sports Med Rehabil 2020; April 27. doi:10.1016/j.asmr.2020.04.008

6. Kim CS, Lynch JB, Cohen S, et al. One academic health system's early (and ongoing) experience responding to COVID-19: recommendations from the initial epicenter of the pandemic in the United States. Acad Med 2020; April 9. doi:10.1097/ACM.0000000000003410

7. O'Reilly GM, Mitchell RD, Noonan MP, et al. Informing emergency care for COVID-19 patients: the COVID-19 emergency department quality improvement project protocol. Emerg Med Australas 2020; 32(3):511-514. doi:10.1111/1742-6723.13513

8. Woolliscroft JO. Innovation in response to the COVID-19 pandemic crisis. Acad Med 2020; April 8. doi:10.1097/ACM.0000000000003402

9. Centers for Disease Control and Prevention, Coronavirus (COVID19). www.cdc.gov/coronavirus/2019-ncov/index.html. Accessed May 20, 2020.

10. World Health Organization. Coronavirus. www.who.int/healthtopics/coronavirus\#tab=tab_1. Accessed May 20, 2020.

11. American Society of Anesthesiologists. COVID-19 information. www.asahq.org/in-the-spotlight/coronavirus-covid-19-information. Accessed May 20, 2020. 